\title{
Alfareros de aquí o de allá: identidad estilística y tecnológica en el valle de Pachacamac (costa central peruana)
}

\author{
Krzysztof MaKowsKi HanUla \\ Pontificia Universidad Católica del Perú \\ kmakows@pucp.edu.pe \\ Gabriela Oré MENÉndeZ \\ Pontificia Universidad Católica del Perú \\ Gabriela.ore@pucp.edu.pe
}

Recibido: 30 de julio de 2013

Aceptado: 10 de septiembre de 2013

\begin{abstract}
RESUMEN
El reconocimiento de identidades étnicas y políticas previas a la conquista española en Perú se ha basado tradicionalmente en los estilos cerámicos. En la costa central, aprovechando la existencia de fuentes sobre la cuenca del Lurín, se ha sugerido que los ayllus costeños se servían preferentemente de la cerámica utilitaria de estilo Ychsma, que definiría también a la cultura del mismo nombre; los habitantes de la cabecera del valle (Huarochirí) habrían utilizado recipientes cerámicos del estilo llamado «Serrano». La frecuente aparición de este segundo estilo en los sitios excavados o prospectados en el valle bajo del Lurín, se ha interpretado como prueba de la presencia e importancia política serrana en la costa. El artículo contrasta esta hipótesis mediante el análisis de Espectrometría de Masas por Ablación Láser con fuente de Plasma de Acoplamiento Inductivo (LA-ICP-MS) de 600 fragmentos de cerámica del valle bajo, medio y alto del Lurín y 116 muestras de arcilla de los valles del Chillón, Rímac, Lurín y Chilca, procesando los resultados mediante estadística multivariante y análisis espacial (SIG) con objeto de correlacionar conjuntos de alfares y grupos estilísticos con las áreas de extracción de la materia prima. Los resultados finales muestran que el valle del Lurín y el valle del Rímac tuvieron cada uno su propia tradición estilística y tecnológica en las vasijas de uso utilitario; indican, asimismo, que el hipotético «Estilo Serrano» caracteriza a la producción de alfareros del valle del Lurín, mientras que el estilo Ychsma se produce principalmente en el valle del Rímac. Estas conclusiones obligan a reinterpretar las relaciones entre sierra y costa durante el Horizonte Tardío, incluyendo el comienzo del Periodo Colonial, y a someter a crítica la existencia de la «cultura Ychsma» en el territorio del hipotético señorío del mismo nombre.
\end{abstract}

Palabras clave: Estilos cerámicos, producción cerámica, arqueometría, LA-ICP-MS, valle del Lurín, Pachacamac, incas.

\section{Potters from Here or There: Stylistic and Technological Identity in the Pachacamac Valley (Peruvian Central Coast)}

\begin{abstract}
The recognition of ethnic and political identities in pre-Conquest Peru has been traditionally based on ceramic styles. In the Central Coast, taking advantage of the existence of historical sources about the Lurin Valley, it has been suggested that coastal ayllus were using preferably Ychsma Style utilitarian pottery, which would also define the culture of the same name; the inhabitants of headwaters of the valley (Huarochirí) would have used so-called «Serrano» Style pottery. The frequency of occurrence of this style in excavated or surveyed sites at Lower Lurin Valley has been interpreted as evidence for highland presence and political importance on the coast. The article tests this hypothesis by analyzing, through Laser Ablation Inducted Couple Plasma Mass Spectrometry (LA-ICP-MS), 600 potsherds from Lower, Middle and Upper Lurin Valley and 116 clay samples from Chillon, Rimac, Lurin and Chilca valleys; the results has been processed by multivariate statistics and spatial analysis (SIG) in order to correlate ensembles of pottery workshops and stylistic groups with areas of clay extraction. The final results show that the Lurin Valley and the Rimac Valley each had its own stylistic and technological tradition in utilitarian pottery; they also show that the hypothetical «Serrano» Style was characteristic of potters in the Lurin Valley, while Ychsma Style was mainly produced in the Rimac Valley.
\end{abstract}


Such findings force us to reinterpret the relationship between highlands and coast during the Late Horizon, including the early Colonial Period, and to subject to criticism the existence of the «Ychsma culture» in the territory of the hypothetical chiefdom of the same name.

Key words: Ceramic styles, pottery production, archaeometry, LA-ICP-MS, Lurin Valley, Pachacamac, Inca.

Sumario: 1. Introducción. 2. Estilos e identidad estilística. 3. Materia prima e identidad tecnológica. 4. Definición de las identidades tecnológicas a partir del estudio arqueométrico. 5. Resultados en la cerámica y en la arcilla. 6. Conclusiones. 7. Referencias bibliográficas.

\section{Introducción}

En la arqueología de la costa central del Perú prehispánico, como en muchas otras partes de los Andes Centrales, las cronologías relativas, las reconstrucciones de espacios políticos y fronteras en la prehistoria, y asimismo, de identidades étnicas, políticas o religiosas, se fundamentan en la definición de un estilo de cerámica a partir de criterios de acabado de superficie y de repertorio de formas. Se asume asimismo que este estilo es exclusivo o por lo menos dominante, y por lo tanto recurrente de manera uniforme en ciertos periodos y espacios en los que coincide con ciertos estilos de arquitectura y textiles, de artefactos de madera, mate pirograbado o metales, siempre y cuando estos últimos se conserven. Detrás de las cronologías estilísticas y procesuales actualmente en uso está un modelo de desarrollo cuyos cimientos se encuentran ya en los trabajos pioneros de Uhle (Shimada 2010: 18). En aquel modelo, los periodos de desarrollos locales se alternan con otros en los que estilos influyentes supuestamente integran a los Andes Centrales como región o como un área de «co-tradición». De acuerdo con estas premisas, los investigadores de ayer y hoy han estado buscando definir un estilo local que sucedería en el tiempo al fenómeno integrador huari (Horizonte Medio) y antecedería a otra integración, esta vez provocada por la expansión del Imperio Inca. En el caso de la costa central, se conocía bien desde el siglo XIX el estilo muy particular de cerámica, difundido en el valle del Chancay y presente también en Ancón. La situación en los otros tres valles en cuya desembocadura se extiende hoy la ciudad de Lima quedaba por definirse. Muy recientemente se ha propuesto llamar al estilo regional de la cerámica del Periodo Intermedio Tardío, la que se producía en los valles del Lurín, Rímac y Chillón, con el vocablo Ychsma siguiendo la propuesta de Bazán (1990) y Vallejo $(2004,2009)$ (Feltham y Eeckhout 2004).

Los autores arriba mencionados han hecho suya la hipotética reconstrucción de las fronteras étnicas y políticas propuesta años atrás por Rostworowski (2002 inter alia) para la costa central del Perú. Según la influyente historiadora, ni el Inca Pachacuti ni su sucesor Inca Túpac Yupanqui habían intentado avasallar por la fuerza al señorío costeño en cuyo territorio se hallaba el santuario y oráculo de Pachacamac; la razón de esta benevolencia habría sido el supuesto respeto a la famosa deidad. Por ende, los límites y la organización de la provincia del Tahuantinsuyo -el huamani de Pachacamac- habrían conservado sin cambio alguno la organización y las fronteras originales del señorío regional, el que, se supone, había surgido siglos atrás a raíz de la descomposición del imperio Huari (Díaz 2004; Cornejo 1999, Eeckhout 2009). La historiadora ha sugerido que todo el señorío adoptó el nombre aymara que en diferentes fuentes fue transcrito como Ychsma, Ichma, Isma o Irma, un vocablo que en 
otras fuentes se refiere a un colorante, probablemente el «achiote» (Bixa orellana), a la acción de pintarse el rostro (González Holguín 1608), o al azogue (Calancha 1639; Prado 1974: 842; Santacruz Pachacuti Yamqui 1968 [1613]: Fol. 1298; Vázquez de Espinoza 1992 [1629]). No obstante, no hay pruebas inequívocas sobre el escenario político señalado por la historiadora. En las fuentes, la voz Ychsma, Ychima (Albornoz 1967 [1569]: 34), Ichma o Irma se refiere únicamente al antiguo nombre del valle del Lurín (Castro y Ortega Morejón 1934 [1558]), sustituido luego por los incas por el nombre del oráculo: «... y así se mudó el nombre del dicho valle de Irma Pachacamacamahc» (Santillán 1968 [1553]: 111). Por extensión, el nombre del río se convirtió en la denominación de las comunidades -parcialidades-ayllus- asentadas en ambas orillas del valle bajo del Lurín, como consta en los documentos del año 1580, y también sobrevivió en los nombres propios de varios principales, tanto de Pachacamac como de Amacas (Rostworowski 2002: 75). La implantación forzosa del asentamiento de mitmaquna militares en Pueblo Viejo-Pucará y el impresionante volumen de construcciones emprendidas por los incas en Lurín y en Rímac, transformando por completo su organización política (Makowski 2002; Makowski et al. 2008), no concuerdan bien con el hipotético escenario de la incorporación pacífica del territorio de la costa central al Tahuantinsuyo propuesta por Rostworowski. En ausencia de evidencias contundentes de que el vocablo Ychsma se hizo también extensivo al señorío y a los habitantes de los valles del Rímac y Chillón, la supuesta existencia de lazos de etnicidad compartida y fortalecida por la integración política en las tres cuencas vecinas merece una reconsideración. El propósito del presente artículo es contribuir a la revisión de las hipótesis fundamentadas únicamente en las escasas fuentes etnohistóricas, a partir del estudio del sistema de producción alfarera en la costa central del Perú durante el Horizonte Tardío.

\section{Estilos e identidad estilística}

Como se ha visto, los tradicionales enfoques del tema del ethnos y de la cultura siguen ejerciendo una fuerte influencia sobre la arqueología de la costa central. En estos enfoques «...la reconstrucción del pasado, tributaria de una definición particular de la cultura arqueológica, se teje en el escenario de culturas homogéneas cuyas historias siguen narrativas lineales en las que secuencias de eventos objetivos, como contactos, migraciones y conquistas, se miden por intervalos de tiempo homogéneo y continuo que separan un evento del otro... Este tipo de marco cronológico es el que Fabian (1983: 23) ha caracterizado como tiempo tipológico» (Jones 1996: 64; trad. $\mathrm{K}$. Makowski). En este sentido, muchos autores esperaban que, en el valle del Lurín, el conflicto latente entre los pobladores del valle medio y alto (Huarochirí) y los del valle bajo (Ychsma), por un lado, y entre los pastores de las lomas y los agricultores y pescadores del valle, por el otro, se reflejara directamente en el cambio de fronteras de distribución de estilos cerámicos supuestamente característicos de cada uno de estos grupos.

Es útil recordar, siguiendo a Trigger (1989: 155-174) y Renfrew (1996: 126-128), que la acepción de la cultura como el espacio de difusión uniforme de un estilo ex- 
clusivo de un grupo étnico en particular se origina en el calor del debate sobre los orígenes de la civilización occidental. Las obras y los puntos de vista de Kossinna y de Childe han ejercido una influencia decisiva sobre ella. Para Childe (1940: 2) «la prehistoria es capaz de reconocer a los pueblos y los maneja de tal manera que éstos se constituyen en actores comparables con los personajes que conforman la compañía del historiador». Por otro lado, como bien observa Jones (1996: 64; trad. K.Makowski), «las expectativas de que las culturas sean siempre territorialmente continuas, homogéneas y posean fronteras definidas se manejan desde el siglo XIX en relación con el nacionalismo y con el estado-nación emergente». Profundos cambios han acontecido en la manera de percibir el problema de la identidad étnica en arqueología y antropología a partir de los años sesenta y al inicio de los setenta del siglo pasado, cuando proliferaron las investigaciones sobre la etnicidad y sobre la hipotética relación entre las fronteras étnicas y estilísticas en la arqueología y la prehistoria (Jones 1996: 66; Dragadze 1980; Renfrew 1996: 130). Para Barth (1969: 19), cuyo aporte a la discusión es particularmente influyente y significativo, la identidad de los grupos étnicos no se desprende del listado de similitudes y diferencias formuladas por el investigador, sino de categorías de adscripción y de identificación que establecen los mismos actores sociales. Se trata de un subjetivo «we-feeling» (Francis 1947: 397) que puede ser abordado sólo de la perspectiva emic, la del participante, en lugar de etic, la del observador (Renfrew 1996: 130). De ser así, habría que darle la razón a Fardon (1987: 176) en que la arqueología no sólo ha sido incapaz de demostrar la validez real de sus criterios de reconstrucción de identidades sociales en la prehistoria, sino que simplemente los ha inventado. Por consiguiente, el tema quedaría vetado para la arqueología. No obstante, los aportes de la reflexión estructural en materia de la identidad tecnológica (chaîne opératoire), de los comportamientos funerarios y de la organización del espacio doméstico, así como la teoría post-estructural de Bourdieu, han abierto nuevos derroteros.

Vista desde la perspectiva de la teoría de la práctica de Bourdieu (1977), «la construcción subjetiva de la identidad étnica en el contexto de la interacción social se funda en la compartida y subliminal disposición del habitus, el que adquirió su forma y sigue siendo formado por las prácticas comunitarias» (Jones 1996: 68). «El habitus compartido engendra sentimientos de identidad entre la gente con experiencias de vida similares. Estos sentimientos apropiados de manera consciente se materializan y adquieren formas de expresión por medio de recursos simbólicos existentes» (Bentley 1987: 173). Las identidades se construyen y reconstruyen en el constante discurso interno y con el exterior:

«Las categorías en las que descansa la identidad étnica se producen, reproducen y transforman en aquel nivel discursivo por medio de la comunicación social de la diferencia y en relación con las prácticas culturales de los étnicos otros.... De ahí, las configuraciones de la etnicidad y, por consiguiente, el estilo de la cultura material implicado en la expresión del significado y en la estructuración de las relaciones étnicas, puede variar en diferentes contextos sociales y en relación con distintas formas y escalas de interacción social. Desde el punto de vista arqueológico, ello resulta probablemente en patrones sumamente complejos, con una distribución traslapada de componentes de cultura material, en lugar de formar unidades culturales monolíticas. ... Cuando se sistematiza y se racionaliza la distribución de los estilos distintivos en el 
proceso de reconocimiento de la expresión y de la negociación de la identidad étnica, el resultado probablemente adoptará la forma de una distribución discontinua no aleatoria, como han sugerido Hodder (1982) y Wiessner (1983)» (Jones 1996: 72; trad. K. Makowski, énfasis añadido).

Como se desprende de la discusión presentada, ni los «Ychsma» ni los «Caringa» de las fuentes etnohistóricas deberían ser considerados necesariamente como grupos étnicos consolidados y establecidos siglos antes de la conquista inca, como lo sugieren Rostworowski (2002) y Eeckhout (2009). Sus identidades políticas (como parcialidades de un curacazgo valluno) y eventualmente étnicas se forjaron primero a raíz de la convivencia forzada con otros grupos, varios de ellos posiblemente desplazados como mitmaquna. Este fue probablemente el origen de los caringas de Huarochirí. No menos tormentoso y decisivo para la afirmación de identidades viejas y la negociación de nuevas debió ser el periodo de las guerras civiles que siguieron a la llegada de Pizarro (Salomon y Grossboll 2009). No hay que olvidar que la mayoría de fuentes proviene de la segunda mitad del siglo XVI. Cien años habían transcurrido desde la conquista inca y el recuerdo de la organización política anterior debió ser distorsionado por el pasado inmediato y amoldado a los nuevos intereses de la población indígena. Makowski (2002) cree que el mismo nombre de caringas es de origen colonial. El despoblamiento dramático de la costa y el abandono del asentamiento principal de Pueblo Viejo-Pucará probablemente contribuyó a la distorsión de las tradicionales jerarquías de poder en el mundo indígena. El peso de los curacas del litoral había disminuido a favor de los curacas de valle adentro y de lomas, que tuvieron bajo su mando un mayor número de contribuyentes. De ahí la nueva importancia de una zona periférica de caringas en la cuenca del Chilca.

Por otro lado, las identidades étnicas en el contexto de una sociedad tan compleja como la inca o la colonial temprana no tienen por qué reflejarse directamente en los estilos de la cerámica decorada. Estos últimos pueden más bien indicar la amplitud de contactos sociales y la posición política negociada y ostentada por los usuarios. Es de esperar más bien que la identidad étnica se exprese en la organización de espacios domésticos, en el modo de construir la estructura habitacional, en las preferencias en cuanto a la ubicación de asentamientos y de lugares sagrados, en las formas y estilos de vasijas que sirven para preparar y almacenar la comida, en las modalidades de entierro, etc. El estilo Ychsma fue definido en buena parte, como por ejemplo en la fase Ychsma Medio y Tardío A (Vallejo 2004), a partir de formas utilitarias, ollas y cántaros. En este sentido, podría contribuir a la definición del habitus compartido por los grupos asentados en la costa central. No obstante, en todos los asentamientos excavados del Horizonte Tardío en el valle del Lurín, este estilo no es ni único ni tampoco necesariamente mayoritario. Hay otro estilo tan recurrente como Ychsma, a pesar de que ambos se caracterizan por el mismo repertorio de formas esenciales de la cerámica utilitaria, usada para almacenar y cocinar líquidos y sólidos. A partir de las prospecciones de Patterson (1966) y Feltham (1984), generaciones de investigadores consideraron que este segundo estilo caracterizaba a la producción de los alfareros serranos. La alta popularidad de aquel estilo de cerámica utilitaria, de color marrón oscuro en el asentamiento de los mitmaquna procedentes de la sierra de Huarochirí, 
Pueblo Viejo-Pucará (Makowski 2002, Makowski y Vega Centeno 2004), parecía corroborar la validez de esta hipótesis.

\section{Materia prima e identidad tecnológica}

En el presente estudio hemos tomado como punto de partida la coexistencia de dos estilos de cerámica utilitaria equivalentes en uso: uno de ellos, el Ychsma, supuestamente definiría en el tiempo y en el espacio al estilo de vida de la población autóctona, costeña, hipotéticos súbditos de los «señores Ychsma» que se sometieron sin resistencia a los invasores incas de acuerdo con la hipótesis de Rostworowski (2002); el otro estilo correspondería a la vajilla de sus vecinos de la sierra. Habría que esperar, por ende, que el análisis ceramológico fino de las cadenas operativas, correspondientes, respectivamente, a cada uno de los dos estilos, revelaría una identidad tecnológica diferente para cada caso, dado el contexto social potencialmente distinto en el que se desenvolvían los alfareros. La elección de la técnica de manufactura, la forma de quemar, las herramientas utilizadas y la elección de las materias primas son decisiones que reflejan una manera particular de hacer las cosas. Los estudios etnográficos y etnoarqueológicos (Stark 2003) han puesto en evidencia la gran variedad de técnicas y procedimientos aún en uso por los ceramistas tradicionales de los Andes (P. Arnold 2000; Costin 2000; Druc 2001; Druc et al. 2001; Druc 2005; Ramón 1999; Stark 2003). La cadena de decisiones parte por supuesto de la elección de una o varias fuentes de materia prima. La reconstrucción de esta primera parte del proceso de producción se topa con varias limitaciones y condicionamientos de orden natural y cultural. Ni las arcillas ni los temperantes son elegidos al azar. La elección de la fuente o de las fuentes de arcilla involucra un proceso largo de experimentación y exploración. Una de las variables más importantes es la calidad del recurso. Una vez ubicadas las fuentes de arcilla apropiadas, es necesario pasar por un proceso de selección de acuerdo con el tipo de producto que se desea elaborar. Los artesanos producen diferentes tipos de vasijas y para cada uno de ellos emplean determinadas fuentes y combinaciones de arcillas específicas (Costin 2000).

La distancia del recurso también influye en su elección. Arnold (1985) discute las variables involucradas en la elección de la materia prima sobre la base de la disponibilidad de la arcilla y la distancia entre la fuente y el artesano. La ubicación de la cantera de arcilla debe de ser ideal en términos de esfuerzo y calidad del recurso. La energía invertida en el traslado y la recolección debe de ser equitativa con la calidad y la abundancia del recurso. La fuente debería ubicarse dentro del territorio explotable de determinada comunidad. Según Browman (1976), la distancia y el costo asociado de extraerla tienen cuatro componentes:

1) distancia geodésica (distancia entre dos puntos),

2) tiempo necesario para cubrir una determinada distancia teniendo en cuenta los accidentes geográficos,

3) costo del desplazamiento, tanto del artesano como el de movilizar la arcilla extraída, 
4) el costo social, referido al tiempo que el artesano estará fuera de la comunidad y no realiza otras actividades.

Las observaciones de Browman son particularmente interesantes, puesto que se refieren a la organización de la producción de ceramistas tradicionales que se desenvuelven en el seno de las sociedades pastoriles de Junín, área relativamente cercana a Huarochirí. Arqueológicamente es muy difícil definir con precisión qué fuentes de arcilla fueron empleadas en la producción de un determinado tipo de vajilla. En todos los casos etnográficos la arcilla se recoge localmente o en el lugar de origen del artesano (Ramón 2011). Algunas veces los artesanos salen de sus localidades de origen y producen vasijas en sus lugares de destino. No obstante, el transporte de materia prima a distancia es costoso en términos de esfuerzo y, por lo general, los alfareros prefieren abastecerse en lugares no muy distantes de su residencia permanente o de sus residencias temporales. Varios artesanos suelen interactuar en una misma zona de extracción de arcilla.

El método tradicional usado en arqueología para determinar el hipotético origen de la producción cerámica se basaba en el criterio de abundancia, aplicado luego de haber definido el repertorio de estilos, alfares o tradiciones tecnológicas. La clase (estilo) de cerámica más abundante en un yacimiento arqueológico representa a la cerámica local, mientras que los otros taxones minoritarios son foráneos.

Durante el siglo XX se han desarrollado dos grupos de metodologías científicas que permitieron contrastar de manera plausible las hipótesis arqueológicas acerca del origen de los estilos: análisis químicos y mineralógicos. Muchos investigadores prefieren utilizar ambas técnicas de manera complementaria. En nuestras investigaciones se ha realizado también un estudio mineralógico preliminar con el fin de establecer alfares por medio de la caracterización de las pastas. Sin embargo, se ha asumido a título de hipótesis que la caracterización química puede dar resultados relevantes acerca de la distribución geográfica de las áreas de procedencia de arcilla sin que estos sean necesariamente confrontados con los estudios petrográficos convencionales sobre láminas delgadas.

El análisis químico de la cerámica da como resultado las concentraciones de ciertos elementos químicos en cada muestra analizada. Este resultado puede, luego de un proceso estadístico, llevar a agrupar fragmentos químicamente similares. A su vez, es posible identificar las mismas características químicas en las fuentes de arcilla. Ambos procesos permiten vincular las muestras de cerámica analizada con una muestra de arcilla procedente de un lugar específico. Este tipo de análisis es una caracterización química.

La interpretación de los resultados obtenidos de una caracterización química está condicionada por una serie de supuestos que conforman el «postulado de procedencia».

El postulado de procedencia estipula que «existen diferencias químicas entre las fuentes de materia prima, y que el enfoque analítico (químico) puede reconocer estas diferencias. Un corolario sostiene que la variación composicional dentro de una misma fuente es menor que la variación entre las diferentes fuentes» (Bishop et al. 1982). Es decir, las diferencias que podemos identificar entre las muestras sometidas al análisis y, por cierto, dentro de cada una de ellas son menores que las variaciones 
entre cada fuente de materia prima; por lo tanto es plausible asignar vínculos entre las muestras y las fuentes dado su grado de similitud.

Pollard y Heron (2008) hacen recordar con razón que es sumamente difícil encontrar las fuentes precisas utilizadas por los alfareros de antaño y que varios factores pueden alterar la composición química de la materia prima a lo largo del proceso de elaboración y quema del recipiente. Las eventuales diferencias entre la composición del producto final y la de la hipotética fuente de materia prima se deben a:

1) la variabilidad natural de los depósitos de arcilla;

2) la selección y la mezcla de arcillas de diferentes fuentes para obtener el producto adecuado y que mejor se comporte durante el proceso de producción;

3) la remoción de impurezas por medio de la levigación ${ }^{1}$ para obtener la textura deseada;

4) el ciclo del quemado que puede afectar la composición, pues algunos elementos son volátiles; y por último

5) el efecto de procesos posteriores al depósito en los materiales, que pueden haber experimentado alteraciones químicas.

Para evitar errores en la interpretación como resultado de las causas arriba mencionadas es necesario comparar la cerámica con muestras procedentes de talleres o que tengan un origen conocido. Este escenario parece poco prometedor, sobre todo porque son pocos los talleres registrados arqueológicamente; no hemos encontrado, para el caso del valle del Lurín, un área de producción que pueda proporcionarnos aquellos datos faltantes. A pesar de esto es viable confiar en los análisis composicionales, ya que tienen la particularidad de detectar elementos trazas y ultra trazas, y es poco probable que estos elementos representen adiciones deliberadas y que puedan reflejar erróneamente la huella geoquímica de la fuente de arcilla (Pollard y Heron 2008).

El análisis químico exitoso tiene por objetivo definir el patrón de la composición química para cada alfar reconocido. Este patrón se deriva de una manera particular de mezclar y preparar la arcilla. Las variaciones que se presenten en la práctica y durante la experimentación son tan bajas que no significarán un cambio en la identificación del patrón propuesto (Mommsen 2004). Es más que probable que el patrón definido para cada alfar sea único y se puede identificar fácilmente en otros contextos. Es por este principio que, una vez que se identifican los patrones para la cerámica, se puede rastrear el mismo patrón en las muestras de arcilla.

\section{Definición de las identidades tecnológicas a partir del estudio arqueométrico}

\subsection{Análisis arqueométrico}

Los análisis arqueométricos en su aplicación a la cerámica pueden responder a una amplia gama de preguntas de investigación concernientes al uso, a la procedencia y a las modalidades de producción por medio de la descripción cuantitativa y cualitativa de la composición y de la estructura. Para tal fin, la arqueometría analiza las pro-

${ }^{1}$ La levigación es un proceso físico utilizado en la separación de partículas de acuerdo con su granulometría. 
piedades físicas, mecánicas, térmicas, estructurales, químicas y mineralógicas (Rice 2005).

Dentro de los métodos arqueométricos, los análisis mineralógicos o químicos apuntan a definir diferentes propiedades de la cerámica. Sin embargo hay una serie de consideraciones a tener en cuenta antes de elegir un método de análisis complejo. Debemos definir si se analizará un mineral o compuesto, o un grupo o elemento químico en particular. También hay que considerar si los resultados que necesitamos serán cualitativos o cuantitativos. La mineralogía se centra en los componentes cristalinos grandes (inclusiones naturalmente presentes en la arcilla o añadidas después); por otro lado, los análisis químicos pueden definir componentes derivados de elementos presentes en la cerámica. No creemos que exista una oposición entre los diferentes métodos de caracterización cerámica; la mayoría de estos son métodos complementarios.

La Espectrometría de Masas por Ablación Láser con fuente de Plasma de Acoplamiento Inductivo (LA-ICP-MS) ha sido la técnica elegida para el análisis cerámico de nuestras muestras. Mommsen (2004) sugiere que debemos de tener en cuenta una serie de factores presentes en los estudios de caracterización cerámica, en especial cuando usamos caracterización química:

1) Cualquier fragmento de cerámica que se analice debe ser representativo de toda la vasija. Si no fuera este el caso y nos encontramos con un fragmento con una alta concentración de inclusiones o que contiene diferentes elementos traza, esto no generará error en nuestro análisis, ya que será un dato aislado que se corregirá automáticamente durante la aplicación del método completo.

2) Para poder hacer comparaciones satisfactorias y más exactas es necesario que el nivel de precisión de nuestro análisis sea capaz de detectar como mínimo 20 elementos traza. Cualquier análisis con menos variables no permitirá una adecuada separación de grupos químicos significativos. Adicionalmente y en la misma línea, es necesario que el nivel de precisión sea muy alto.

\subsection{Espectrometría de Masas por Ablación Láser con fuente de Plasma de Acopla- miento Inductivo (LA-ICP-MS).}

LA-ICP-MS (por su nombre en inglés: Laser Ablation Inducted Couple Plasma Mass Spectrometry) se desarrolló en los años ochenta a partir de la ICP-MS como uno de los métodos caracterizados como Microsonda. La ICP-MS es una técnica que vaporiza e ioniza una muestra líquida por medio del gas argón. Al formarse los iones, estos son transportados a un espectrómetro de masas donde se identifican y cuantifican. El análisis por ICP-MS requiere que las muestras sean disueltas en ácidos o a altas temperaturas para tener un complejo líquido. Sin embargo se trata de una tarea que consume demasiado tiempo y resulta poco agradable (Neff 2003; Speakman y Neff 2005; Speakman et al. 2007). Es a mediados de los años ochenta cuando al ICP-MS se le añade un láser que «hace posible la caracterización individual de diferentes componentes dentro de matrices heterogéneas, o únicamente la superficie de diversos materiales» (Neff 2003: 21). El bajo costo de análisis, el daño imperceptible ocasionado a las muestras y su sensibilidad para identificar un mayor rango de elementos 
químicos la ha convertido en una de las técnicas más innovadoras y difundidas entre los investigadores de ciencia de materiales en los últimos veinte años (Speakman et al. 2007).

En el ámbito arqueológico este método es el más rápido y sencillo para generar datos composicionales y, asimismo, el menos costoso. En los Andes se ha demostrado que los análisis de cerámica mediante LA-ICP-MS llevan a resultados coincidentes con los que se han logrado mediante la aplicación de otros métodos: por ejemplo en los casos de cerámica Wari (Dussubieux et al. 2007) y de la producción alfarera del Horizonte Tardío en el valle del Lurín por Makowski y Ghezzi (2007). Adicionalmente, como ya hemos mencionado, LA-ICP-MS tiene la facultad de centrarse, para el caso de una muestra de cerámica, en únicamente la matriz de arcilla o exclusivamente en el temperante, pudiendo definir así las características de una de las materias primas en particular.

Una vez analizadas las muestras, los resultados deben pasar por un proceso cuantitativo para normalizar y estandarizar los resultados. La estandarización de muestras líquidas permite usar estándares internos que corrijan la desviación del instrumental empleado; sin embargo, es necesario el uso de una metodología de estandarización diferente para el caso de las muestras usadas por LA-IC-MS. En ese caso es necesario asumir que un elemento que se comporte de manera independiente puede servir de estándar interno (Neff 2003).

Otra manera de estandarizar la muestra y corregir posibles errores es medir algunos de los compuestos más grandes con alguna otra técnica como XRF o incluso INNA. La medición obtenida normaliza los datos arrojados por LA-ICP-MS. Gratuze et al. (2001) presentan un método de normalización que involucra el uso de materiales de referencia (usan materiales provenientes del National Institute of Standards and Technology). Uno de los materiales usados para la estandarización de las muestras es el Ohio Red Cay estandarizado con la muestra SRM612 de NIST (Neff 2003).

Los resultados de los análisis usualmente son expresados en concentraciones en partes por millón en tablas simples. Para poder traducir esta información en datos que apoyen nuestras hipótesis es necesario realizar una serie de rutinas de estadística multivariante para determinar, por ejemplo, grupos químicos.

El uso de LA-ICP-MS permite analizar, como mencionamos líneas arriba, exclusivamente la pasta de las muestras cerámicas, dejando de lado las inclusiones y el engobe o la pintura. Las características químicas de otros elementos, al no ser incluidos, no alteran en nada la definición de los grupos cerámicos. El método de análisis y su consecuente análisis estadístico permiten aislar y ubicar respecto a otras muestras los casos de cerámica producida a partir de una mezcla de varias arcillas diferentes. Una mezcla se reflejará en un grupo químico diferente o en elementos de nuestra muestra que no pueden ser agrupados y se consideran por ende «no asignados».

\subsection{Análisis cuantitativo de los resultados}

Los resultados arrojados por el espectrómetro de masas involucran concentraciones de 45 elementos químicos. Esta cantidad de variables obliga a usar la estadística multivariante y el análisis de componentes principales. 
El primer paso consiste en la exploración de datos. Una vez ordenados y normalizados, se hace uso de diagramas de dispersión y del análisis de componentes principales (ACP) para elegir aquellas variables que agrupan a los datos obtenidos de una mejor manera. El ACP «mide la magnitud y dirección de las variaciones máximas de los datos en el hiperespacio composicional, y genera nuevas variables y valores a partir de las originales. Las nuevas variables, llamadas componentes principales, incorporan la mayor parte de la variaciones en los datos originales» (Ghezzi 2011: 16). Para poder visualizar los datos es recomendable utilizar un «biplot $[\ldots]$ que representa gráficamente la dirección y magnitud con que cada elemento químico contribuye a definir los grupos composicionales (Ghezzi 2011: 17). La clasificación de la muestra en grupos se hace utilizando la Distancia de Mahalanobis. Los grupos determinados se comprueban por medio de un Análisis Discriminante.

Terrádez Gurrea (2002) señala claramente que el objetivo de un análisis de componentes principales (ACP) es sintetizar o reducir el número de variables tratando de perder la menor cantidad de información posible. «La idea general es extraer algo que tengan en común diversas variables» (Shennan 1992: 246). Para lograr este objetivo se trabaja con una combinación lineal de las variables originales que adicionalmente serán independientes entre sí.

Sólo se puede aplicar el ACP si las variables de los datos están altamente correlacionadas. Esto es señal de que existen datos que pueden explicar gran parte de la variabilidad de la muestra y otros que son redundantes.

\section{Resultados en la cerámica y en la arcilla}

\subsection{Grupos químicos}

Para sustentar los objetivos trazados y definir desde la perspectiva de la caracterización química las cadenas operativas correspondientes a dos estilos de la cerámica utilitaria, el «Ychsma» y el «Serrano», en el contexto de otros estilos minoritarios, incluyendo los del grupo Inca, analizamos, en el marco del Programa Arqueológico Valle de Pachacamac, más de 40.000 fragmentos procedentes de los sitios arqueológicos de Pueblo Viejo-Pucará, Pachacamac y Huaycán de Cieneguilla y pre-seleccionados por su carácter diagnóstico desde el punto de vista de forma, acabado y decoración. De este análisis se desprenden más de 16 alfares (Makowski y Vega Centeno 2004) que pueden ser agrupados en cinco estilos básicos:

1) Cerámica comparable desde el punto de vista formal y decorativo con la producción de talleres cuzqueños, en particular los aríbalos Cuzco Polícromo A.

2) Cerámica de alfar marrón que tradicionalmente se relaciona con las tradiciones de la sierra, en la región de Huarochirí. Se le conoce usualmente como «Estilo Serrano».

3) Cerámica estilísticamente vinculada con los estilos foráneos no inca del Horizonte Tardío (p.e. Chimú-Inca) y que, además, suele aparecer asociada con los estilos cuzqueños (p.e. Inca asociado) de Strong y Corbett (1943). 


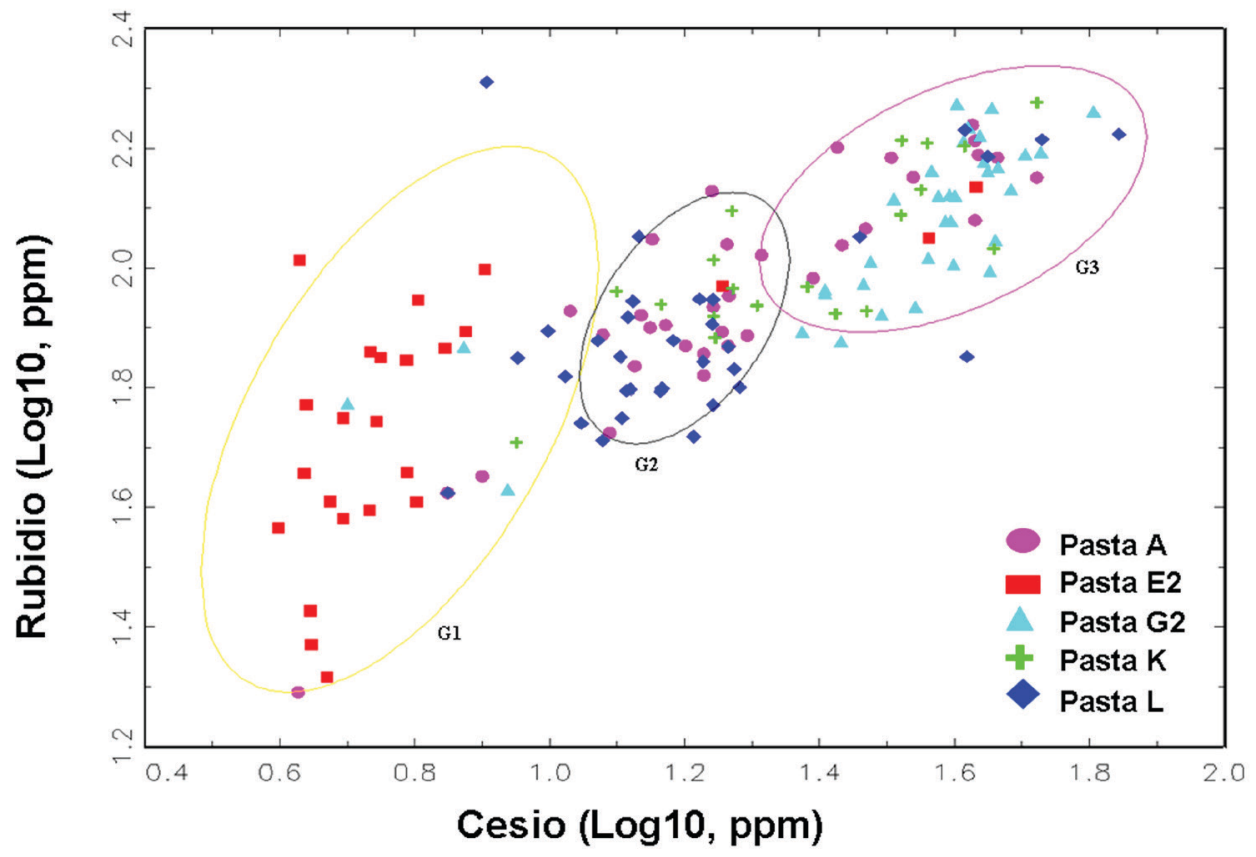

Figura 1: Diagrama de dispersión de la concentración de cesio y rubidio.

4) Cerámica con préstamos estilísticos e imitaciones posiblemente originarios de la costa al sur de Lurín y al norte del Chillón, vg. el estilo Puerto Viejo (Bonavia 1959). Vallejo (2009) la considera una variante decorada del estilo Ychsma.

5) Cerámica que carece de características o préstamos foráneos, salvo los que proceden de los vecinos de la costa central, y que suele encontrarse en contextos posteriores al Horizonte Medio 3. Se propone la denominación Ychsma o Ichma (Bazán del Campo 1990; Vallejo 2004).

Una vez clasificados, se procedió al análisis arqueométrico de muestras correspondientes a cada uno de estos cinco grupos y además de materiales procedentes de prospecciones de la parte media y alta del valle.

El análisis fue hecho en dos etapas. La primera incluyó las muestras provenientes de sitios excavados (Pueblo Viejo-Pucará, Pachacamac y Huaycán de Cieneguilla). Los resultados de los análisis químicos y estadísticos en esta primera etapa revelaron la existencia de tres conglomerados correspondientes a igual número de grupos químicos (Ghezzi y Neff 2008; Makowski et al. 2006; Makowski et al. 2008; Makowski y Ghezzi 2007; Neff 2006). En la segunda etapa se incluyeron datos obtenidos de sitios prospectados en la parte media y alta del valle del Lurín observando su correlación eventual con los grupos químicos previamente definidos.

El análisis composicional respalda los taxones establecidos a partir del estudio macroscópico (Figuras 1 y 2). Por ejemplo, el 91,2\% de las muestras de pasta G2 se asocian con el Grupo 3, mientras que el 87,5\% de las muestras de la pasta E2 corresponden al Grupo 1. Este patrón, en que las muestras de un alfar se asocian con un grupo 


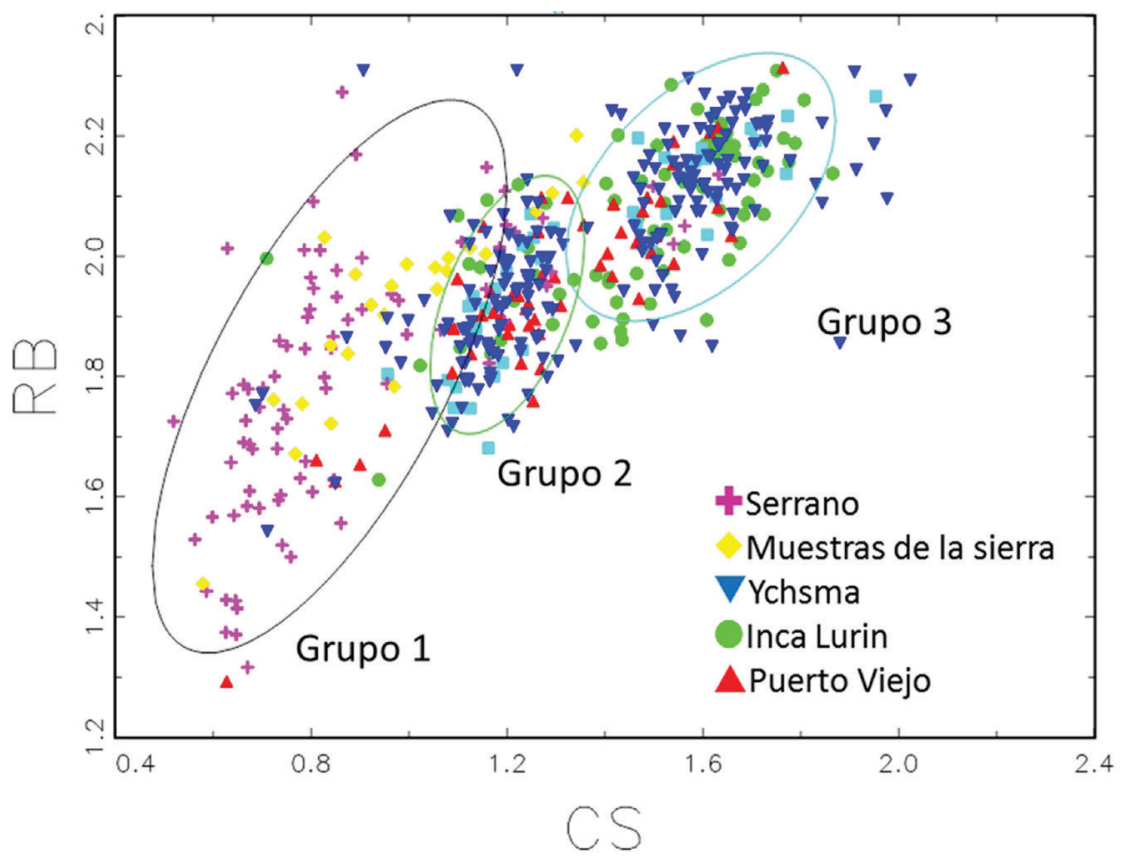

Figura 2: Estilos pertenecientes a los grupos redefinidos.

composicional y tienen una composición homogénea, ilustrada por el agrupamiento entre las muestras, se repite en casi todas las pastas. Por otro lado, una inspección detallada de los datos sugiere que la frecuencia con que se manifiestan los grupos composicionales varía también según la categoría morfo-funcional y grupo estilístico al que la muestra pertenece. Así, el 73,3\% de los fragmentos asignados al Grupo 1 son de estilo Serrano, que prácticamente no se registra en los otros grupos. Los grupos 2 y 3 son más variados estilísticamente, pero también muestran asociaciones específicas. Por ejemplo, un 54,7\% de los tiestos del Grupo 2 es de estilo Ychsma, con los estilos Puerto Viejo, Inca Costeño y Chimú-Inca representados en menores porcentajes. En el Grupo 3, los fragmentos Ychsma e Inca Costeño constituyen el 77,8\%, con los estilos Puerto Viejo y Chimú-Inca presentes en porcentajes pequeños. Un patrón significativo es la oposición en la distribución de los estilos Serrano e Ychsma: cuando uno es abundante, el otro está presente en porcentajes pequeños (Makowski et al. 2011).

GRUPO 1. Al primer grupo químico pertenecen las muestras cerámicas de recipientes utilitarios para almacenar y preparar alimentos con características tradicionalmente consideradas de la sierra y por ende denominadas «Estilo Serrano». La mayoría de los especímenes de cerámica recuperados de la parte alta del valle también forma parte del grupo.

GRUPo 2. El segundo grupo comprende las muestras extraídas de recipientes similares al Grupo 1 en cuanto a las funciones utilitarias y al repertorio de formas, pero confeccionadas en estilos diferentes que se originan en las tradiciones alfareras costeñas (Ychsma e Inca Provincial). 
GRUPO 3. Este grupo comprende mayoritariamente las vasijas en estilo Inca-Lurín, en particular platos y aríbalos. Lo integran también algunas vasijas escultóricas del estilo Ychsma.

En la literatura sobre análisis composicional de cerámica, los grupos identificados generalmente se interpretan como resultado de la explotación de fuentes particulares de arcilla (Mommsen 2001). Desde la perspectiva metodológica de D. Arnold (2000), la principal utilidad del análisis composicional de pastas recae en la identificación de espacios geográficos y geoquímicos de fuentes de explotación comunitarias dentro de un determinado rango de distancia - a los que denomina «community signature units»-. El análisis de las pastas provee de este modo información importante sobre la aparición y desaparición de estas fuentes y adicionalmente sobre el movimiento de la cerámica.

\subsection{Posibles áreas de extracción de arcilla}

Como mencionamos anteriormente, junto con el análisis arqueométrico de la cerámica realizamos una recolección sistemática de arcillas en los valles de la costa central del Perú -Chillón, Rímac, Lurín y Chilca- con el objetivo de tener un mapeo químico de la distribución de arcillas. Las muestras recogidas abarcaron casi todas las quebradas de dichos valles, desde la desembocadura hasta aproximadamente 3.500 m.s.n.m.

Las arcillas colectadas fueron comparadas con los grupos químicos identificados en las muestras cerámicas sometidas al análisis mediante LA-ICP-MS. Los resultados, expresados en la probabilidad de cada muestra de pertenecer a uno de los grupos químicos identificados, fueron mapeados en un SIG (Sistema de Información Geográfica) utilizando la densidad de Kernel. De esta manera fue posible localizar potenciales lugares de procedencia de las fuentes de arcilla utilizadas en la producción de cada grupo (Figura 3).

Las características químicas del Grupo 2 y del Grupo 3 son plenamente coincidentes con las arcillas procedentes del valle del Rímac, donde probablemente se ubicaron también los talleres. La composición química del Grupo 2, que comprende la cerámica Ychsma, caracteriza a las fuentes de arcilla en la zona de Nievería, en el valle del Rímac, un área que hasta la actualidad es un área de extracción preferida para las ladrilleras y las fábricas de terracota. El Grupo 3 relacionado con la cerámica Inca Local, por otro lado, tiene una posible área de producción en la parte baja del valle, en la zona cercana al sitio de Armatambo, un importante centro del Horizonte Tardío. En estos dos últimos casos es muy probable que los talleres se ubicaran cerca de las fuentes de extracción.

En cuanto al Grupo 1, cuyas propiedades químicas caracterizan a la cerámica en el «Estilo Serrano», se han identificado dos posibles áreas de extracción de arcilla, una en la parte baja del valle cerca de la zona de Lúcumo, camino al sitio de Pueblo Viejo, y la otra en el área cercana al sitio de Sisicaya en el valle medio. Estos datos arrojan nueva luz sobre la producción de la cerámica que por mucho tiempo fue considerada foránea en la costa. Las áreas de producción estuvieron sin duda localizadas en el valle del Lurín mismo, en la cercanía de las zonas residenciales de los asentamientos donde se ha registrado la cerámica de «Estilo Serrano» y de donde provienen las 


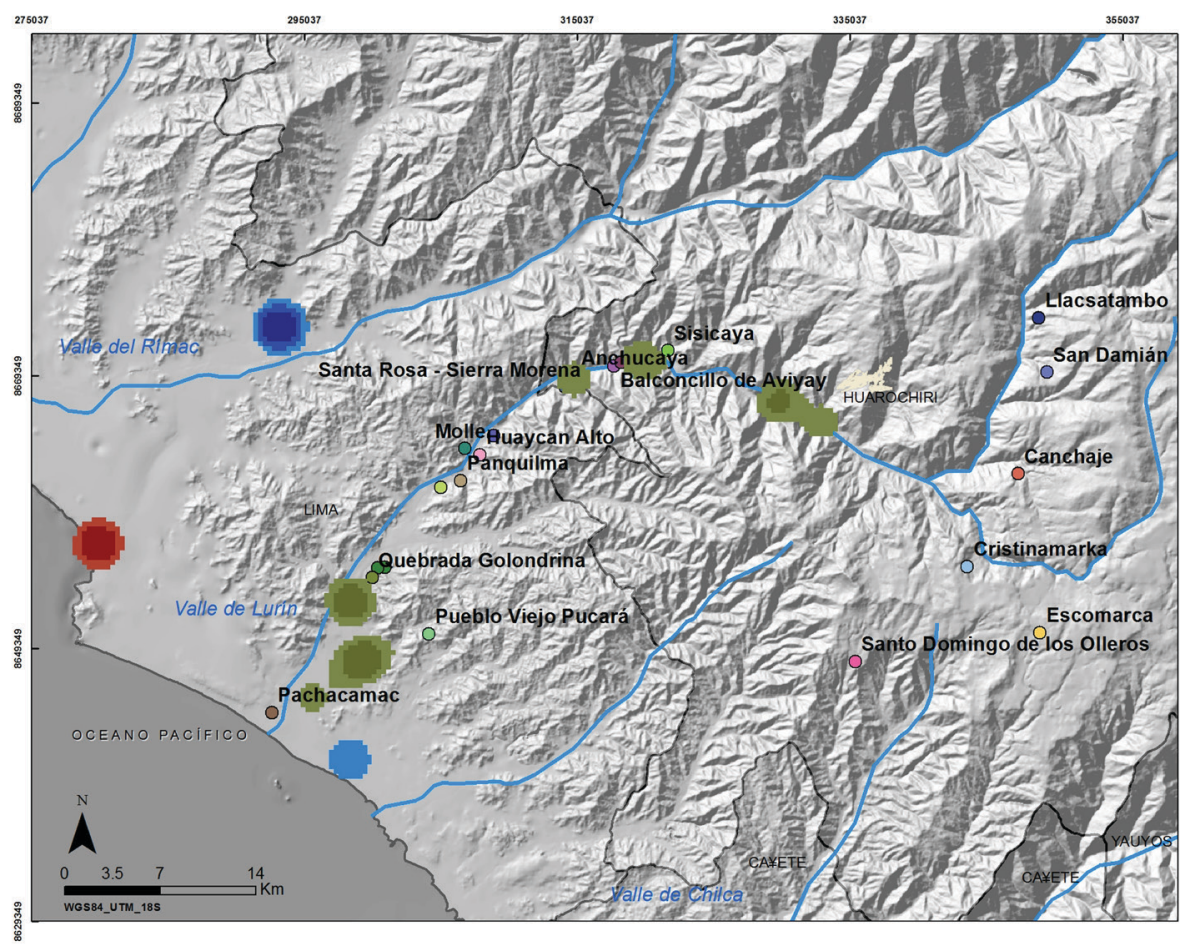

Figura 3: Ubicación de las fuentes de arcilla en la costa central del Perú.

muestras. Los alfareros que abastecían los sitios del valle bajo y también los sitios ubicados en la parte alta compartían algunas de las fuentes de arcilla.

La arcilla cuyos yacimientos se ubican en las lomas de Lúcumo es la que guarda fuerte parentesco con las pastas del «Estilo Serrano». Esta zona se localiza en el centro de un círculo de $20 \mathrm{~km}$ de radio en el cual se ubican los sitios de Pachacamac y Huaycán de Cieneguilla. El sitio de Pueblo Viejo, por otro lado, se ubica sólo a 5 $\mathrm{km}$ de la cantera. Recordemos que Pueblo Viejo es el sitio que presenta mayor recurrencia estadística de la cerámica en el «Estilo Serrano» (alrededor del 40\%); sin embargo, a pesar de haber excavado los cinco sectores, todas las áreas residenciales y dos palacios, cubriendo $14.000 \mathrm{~m} 2$ de área, no se ha registrado ninguna evidencia de producción local de cerámica (Makowski 2002; Makowski y Vega Centeno 2004; Makowski et al. 2008; 2011).

\section{Conclusiones}

Por lo expuesto, existe una relación significativa entre la composición y las dimensiones morfo-funcionales y estilísticas en las series analizadas de muestras de pastas y de arcillas. Los dos estilos correspondientes a la cerámica utilitaria destinada a la cocción y al almacenamiento, el «Ychsma» y el «Serrano», han sido sin duda produ- 
cidos a partir de arcillas procedentes de fuentes diferentes y alejadas unas de otras. En cambio, el Grupo 3, que comprende varios estilos, algunos de ellos foráneos, como el Inca Polícromo o Chimú-Inca, imitados en la costa central, y se define a partir de las vasijas para servir bebidas y comidas, así como piruros, fue constituido para la producción hecha a partir de arcillas de muy alta calidad, que se encuentran cerca de los centros administrativos en la cuenca del Rímac. Coincidiendo con lo señalado por D. Arnold (2000) a partir de observaciones etnográficas, en nuestro caso tampoco se puede establecer una relación directa entre los grupos químicos y la organización social de la producción y redistribución de cerámica en el espacio y en el tiempo. No obstante, el análisis realizado no sólo nos ha acercado a la reconstrucción del mapa de fuentes de arcilla sino también a otros aspectos de la cadena operativa. Los resultados obtenidos han confirmado de manera indirecta la validez de la clasificación en alfares. Recordemos que cada pasta y alfar responde a una tradición tecnológica con variantes definidas en la selección y mezcla de arcillas e inclusiones. Por otro lado, la relación estrecha entre la composición química, las formas relacionadas con el uso y función y los estilos, sugieren además que cada grupo definido corresponde a la tradición tecnológica de un taller o grupo de talleres. Cada taller representa de hecho una tradición tecnológica, expresada en el uso de ciertas fuentes de arcilla; una «receta» de preparación de la pasta, incluidos los temperantes, acorde con la función y tamaño del recipiente, las técnicas de elaboración específicas; los modos de acabado, etc. Ciertos talleres abastecerían grupos o espacios sociales determinados, o responderían a necesidades concretas, por ejemplo la fabricación de vasijas grandes para cocción o almacenamiento. En cualquier caso, el número elevado de alfares-talleres que se especializaban en la producción de los mismos estilos y compartían fuentes de arcilla comunes demuestra que la producción fue descentralizada, como suele ocurrir cuando la elaboración de vasijas no implica el uso de torno alfarero o de molde. Esta conclusión se refuerza por otra observación. Las imitaciones de vasijas de uso ceremonial, a menudo relacionado con ceremonias estatales, como por ejemplo aríbalos y escudillas en el estilo Cuzco Polícromo, no fueron producidas por alfareros que compartían los mismos procedimientos tecnológicos ni tampoco las mismas fuentes de arcilla (Makowski et al. 2008; 2011). La reconstrucción tentativa del sistema de producción y distribución se dibuja por ende de la manera siguiente.

Los alfareros de la parte baja del Lurín y Rímac se organizaban durante el Horizonte Tardío en numerosos talleres o grupos de trabajo. De ahí las diferencias macroy microscópicas en textura, compactación, color y tipo de inclusiones en la pasta. Algunos de estos talleres producían cerámica de estilos exóticos, imperiales, como por ejemplo Inca y Chimú-Inca, además de confeccionar recipientes de características locales. Asimismo, los talleres usaban fuentes de arcilla comunes situadas tanto en el valle del Rímac como el del Lurín. Los alfareros que producían vasijas utilitarias para almacenar y producir alimentos en el estilo denominado Ychsma usaban de manera casi exclusiva las fuentes de arcilla del valle bajo del Rímac, ubicadas cerca de Armatambo y Cajamarquilla (Grupo 2). En cambio, los ceramistas que confeccionaban vasijas equivalentes en uso (Sackett 1986) a las de estilo Ychsma, pero caracterizadas por el estilo que anteriormente llamábamos Serrano, extraían su arcilla de las fuentes situadas en el valle bajo y medio del Lurín, en las cercanías, respecti- 
vamente, de Pachacamac y de Sisicaya (Grupo 1). En ambos casos, llama la atención la proximidad de importantes asentamientos imperiales, considerados centros administrativos inca, y de las canteras de arcilla. La cerámica fina para servir bebidas y comidas, con frecuentes imitaciones de estilos foráneos (Grupo 3), fue producida a partir de la arcilla procedente de los alrededores de Armatambo y de la quebrada de Huachipa. Desafortunadamente, no se ha podido encontrar aún talleres a pesar de las insistentes prospecciones (Agurto y Watanabe 1974; Feltham 1984; Patterson 1966) y numerosas excavaciones. No obstante, estos resultados invitan a reconsiderar las opiniones dominantes en la literatura del tema en cuanto a la presunta relación entre un estilo cerámico y las identidades étnicas y políticas de productores y usuarios. Sin duda, los integrantes de los ayllus denominados Ychsma Caringa (Pachacamac) y Caringa de Huarochirí (Pueblo Viejo-Pucara) (véase Makowski 2002) asentados en las orillas del río Ychsma, rebautizado en los tiempos inca como el valle de Pachacamac, no usaban de manera preferente el estilo Ychsma. Todo lo contrario, el mayor uso lo tuvieron los recipientes en estilo conocido como Serrano y típico para Lurín. Asimismo, tal como lo hemos sugerido ya anteriormente (Makowski et al. 2008), las fronteras étnicas no se expresan en la distribución de estilos de cerámica porque las identidades de productores y usuarios no coinciden. El sistema-mundo inca, no obstante todas sus particularidades en cuanto a la organización de producción y distribución, crea condiciones para que las poblaciones tengan acceso simultáneo a la cerámica en diferentes estilos.

Agradecimientos: Los resultados de investigación reciente que presentamos en este artículo forman parte del proyecto a largo plazo desarrollado en el marco del Programa ArqueológicoEscuela de Campo PUCP-Asociación Atocongo «Valle de Pachacamac» que pudo realizarse gracias a la participación de varios investigadores. El Dr. Hector Neff (California State University Long Beach) junto con el Mag. Ivan Ghezzi (Yale University - Pontificia Universidad Católica del Perú) tuvieron a su cargo el análisis arqueométrico y estadístico. Manuel Lizárraga, Rosabella Alvarez-Calderón, Milagritos Jiménez, Carla Hernández, Zachary Chase y Christopher Milan participaron en diferentes etapas de recolección de datos en campo y en su procesamiento en el laboratorio. El vicerrectorado académico de la PUCP y la Unión Andina de Cementos S.A.A (UNACEM) financiaron las investigaciones. A todos ellos y a las demás instituciones y personas que han contribuido en nuestras investigaciones de manera directa $\mathrm{o}$ indirecta quisiéramos expresar los más profundos agradecimientos.

\section{Referencias bibliográficas}

Agurto, Santiago y Luis Watanabe

1974 Inventario, catastro y delimitación del patrimonio arqueológico del valle bajo del río Lurín. Lima: Archivo del Centro de Investigación y Restauración de Bienes Monumentales del Instituto Nacional de Cultura.

Albornoz, Cristóbal de

1967 «Las instrucciones para descubrir las guacas del pirú y sus camayos y haciendas» [1569], en «Un inédit de Cristóbal de Albornoz», de Pierre Duviols. Journal de la Société des Américanistes 56 (1): 7-39. 
ARnold, Dean E.

1985 Ceramic Theory and Cultural Process. Cambridge: Cambridge University Press.

2000 «Does the Standardization of Ceramic Pastes Really Mean Specialization?». Journal of Archaeological Method and Theory 7 (4): 333-375.

ARnOLD, Philip J.

2000 «Working Without a Net: Recent Trends in Ceramic Ethnoarchaeology». Journal of Archaeological Research 8 (2): 105-133.

BARTH, Fredrik

1969 Ethnic Groups and Boundaries: the Social Organization of Culture Difference. Oslo: Universitetsforlaget.

BAZÁn DEL CAMPO, Francisco

1990 Arqueología y etnohistoria de los periodos prehispánicos tardíos de la Costa Central. Tesis de Licenciatura. Lima: Universidad Nacional Mayor de San Marcos.

Bentley, Carter

1987 «Ethnicity and Practice». Comparative Studies in Society and History 29 (1): 24-55.

Bishop, Ronald L, Robert L. Rands y George R. Holley

1982 «Ceramic Compositional Analysis in Archaeological Perspective», en Advances in Archaeological Method and Theory, Michael B. Schiffer, ed., vol. 5, pp. 275-330. Nueva York: Academic Press.

BonAVIA, Duccio

1959 «Cerámica de Puerto Viejo (Chilca)», en Actas del II Congreso Nacional de Historia del Perú, pp. 137-168. Lima.

Bourdieu, Pierre

1977 Outline of a Theory of Practice. Cambridge: Cambridge University Press.

BRowman, David L.

1976 «Demographic Correlations of the Wari Conquest of Junin». American Antiquity 41 (4): 465-477.

Calancha, Antonio de la

1639 Crónica moralizada del orden de San Augustin en el Peru: con sucesos egenplares en esta monarquia. Barcelona: Pedro Lacavalleria.

Castro, Cristóbal de, y Diego de Ortega Morejón

1934 «Relación sobre el gobierno de los incas» [1558], en Relación del sitio del Cusco y principio de las guerras civiles del Perú hasta la muerte de Diego de Almagro, 1535-1539. Relación sobre el gobierno de los incas. Anotadas y concordada con las crónicas de Indias, edición de Horacio H. Urteaga. Lima.

CHILDE, Gordon

1940 Prehistoric Communities of the British Isles. Londres: W. \& R. Chambers.

Cornejo Guerrero, Miguel

1999 An Archaeological Analysis of an Inka Province: Pachacamac and the Ychsma Nation of the Central Coast. Tesis doctoral. Australian Nacional University, Canberra.

Costin, Cathy L.

2000 «The Use of Ethnoarchaeology for the Archaeological Study of Ceramic Production». Journal of Archaeological Method and Theory 7 (4): 377-403. 
DíAz ArriolA, Luisa

2004 «Armatambo y la sociedad Ychsma». Bulletin de l'Institut Français d'Etudes Andines 33 (3): 571-594.

Dragadze, Tamara

1980 «Soviet Ethnos Theory». RAIN 38: 3-4.

DRuc, Isabelle C.

2001 «Soil Sources for Ceramic Production in the Andes», en Archaeology and Clays, Isabelle C. Druc, ed., pp. 95-106. BAR International Series, 942. Oxford: Archaeopress.

2005 Producción cerámica y etnoarqueología en Conchucos, Ancash, Perú. Lima: Instituto Cultural Rvna.

Druc, Isabelle C., Richard L. Burger, Regina Zamojska y Pierre Magny

2001 «Ancón and Garagay Ceramic Production at the Time of Chavin de Huántar». Journal of Archaeological Science 28: 29-43.

Dussubieux, Laure, Mark Golitko, Patrick Ryan Williams y Robert J. SPEAKman

2007 «Laser Ablation - Inductively Coupled Plasma - Mass Spectrometry Analysis Applied to the Characterization of Peruvian Wari Ceramics», en Archaeological Chemistry, Michael D. Glascock, Robert J. Speakman y Rachel S. Popelka-Filcoff, eds., pp. 349-363. Washington: American Chemical Society - Oxford University Press.

EeckHout, Peter

2009 «Poder y jerarquías ychsmas en el valle de Lurín». Arqueología y Sociedad 19: 223-240. Lima.

FABIAN, Johannes.

1983 Time and the Other: How Anthropology Makes Its Object. Nueva York: Columbia University Press.

FARDON, Richard

1987 «African Ethnogenesis: Limits to the Comparability of Ethnic Phenomena», en Comparative Anthropology, Ladislav Holy, ed., pp. 168-187. Oxford: Basil Blackwell.

Feltham, Jane

1984 «The Lurin Valley Project. Some Results for the Late Intermediate and Late Horizon», en Current Archaeological Projects in the Central Andes, Ann Kendall, ed., pp. 45-73. BAR International Series. vol. 210. Londres: Archaeopress.

Feltham, Jane y Peter Eeckhout

2004 «Hacia una definición del estilo Ychsma: Aportes preliminares sobre la cerámica Ychsma tardía de la pirámide III de Pachacamac». Bulletin de l'Institut Français d'Etudes Andines 33 (3): 643-679.

FRANCIS, Emerich

1947 «The Nature of the Ethnic Group». American Journal of Sociology 52 (5): 393-400.

GHEZzi, Iván

2011 «El análisis composicional en el estudio de la producción y distribución de la cerámica prehispánica». Bulletin de I'Institut Français d'Études Andines 40 (1): 1-29.

GHEZzi, Iván y Hector NeFF

2008 LA-ICP-MS of Ceramics from the Lurin Valley, Peru. Report presented to Krzysztof Makowski-PATL. Facultad de Letras y Ciencias Humanas, Pontificia Universidad Católica del Perú, Lima. 
GonzÁlez Holguín, Diego

1952 Vocabulario de la lengua general de todo el Perú llamada Lengua qquichua o del inca [1608]. Lima: Universidad Nacional Mayor de San Marcos.

Gratuze, B., M. Blet-Lemarquand y J. N. Barrandon

2001 «Mass Spectrometry with Laser Sampling: A New Tool to Characterize Archaeological Materials». Journal of Radioanalytical and Nuclear Chemistry 247 (3): 645-656.

JoNES, Siân

1996 «Discourses of Identity in the Interpretation of the Past», en Cultural Identity and Archaeology: The Construction of European Communities, Paul Graves-Brown, Siân Jones y Clive Gamble, eds., pp. 62-80. Londres: Routledge.

MaKowsKi, Krzysztof

2002 «Arquitectura, estilo e identidad en el Horizonte Tardío: el sitio de Pueblo ViejoPucará, valle de Lurín». Boletín de Arqueología PUCP 6: 137-170.

Makowski, Krzysztof e Iván GHEzzi

2007 Redes de producción e intercambio en el Horizonte Tardio: Caracterización por espectrometría (LA-ICP-MS) y activación neutrónica (INAA) de los estilos cerámicos de Pachacamac, Huaycán de Cieneguilla y Pueblo Viejo (Valle de Lurín). Concurso Anual de Proyectos de Investigación DAI - PUCP.

Makowski, Krzysztof, Iván GHezzi, Hector NefF y Daniel Guerrero

2006 «Late Horizon Pottery and Social Identity in the Lurin Valley: Report on LA-TOFICP-MS Analysis». Ponencia presentada en la 71st Annual Meeting of the Society for American Archaeology, Puerto Rico, 26-30 de abril. http://patl.pucp.edu.pe/ sites/patl.pucp.edu.pe/files/LA-TOF-ICP-MS_0.pdf, con acceso el 30/9/2013.

Makowski, Krzysztof, Iván Ghezzi, Hector NefF, Daniel Guerrero, Milagritos Jiménez y Gabriela ORÉ

2008 «Pachacamac, Ychsma y los Caringas: estilos e identidades en el valle de Lurín Inca», en Arqueología de la costa centro sur peruana, Omar Pinedo y Henry Tantaleán, eds., pp. 267-307. Lima: Avqui Ediciones.

Makowski, Krzysztof, Iván Ghezzi, Hector Neff y Daniel Guerrero, Milagritos Jiménez, Gabriela Oré y Rosabella Álvarez CALDERón

2011 «Redes de producción e intercambio en el Horizonte Tardío: Caracterización con LATOF-ICP-MS e INAA de arcillas y estilos cerámicos en la costa central del Perú», en II Congreso Latinoamericano de Arqueometría, Luisa Vetter, Rafael Vega-Centeno, Paula Olivera y Susana Petrick, eds., pp. 263-274. Lima: IPEN - UNI - OEI.

Makowski, Krzysztof y Milena Vega Centeno

2004 «Estilos Regionales en la Costa Central en el Horizonte Tardío Una aproximación desde el valle de Lurín». Bulletin de l'Institut Français d'Etudes Andines 33 (3): 681-714.

Mommsen, Hans

2001 «Provenance Determination of Pottery by Trace Element Analysis: Problems, Solutions and Applications». Journal of Radioanalytical and Nuclear Chemistry 247 (3): 657-662.

2004 «Short Note: Provenancing of Pottery- The Need for an Integrated Approach?». Archaeometry 46 (2): 267-271. 
NeFF, Hector

2003 «Analysis of Mesoamerican Plumbate Pottery Surfaces by Laser Ablation-Inductively Coupled Plasma-Mass Spectrometry (LA-ICP-MS)». Journal of Archaeological Science 30 (1): 21-35.

2006 LA-ICP-MS of Ceramics from the Lurin Valley, Peru. Report presented to Ivan Ghezzi-PATL. Dirección Académica de Investigación. Pontificia Universidad Católica del Perú, Lima.

Pachacuti Yamqui Salcamaygua, Juan de Santa Cruz

1968 «Relación de antigüedades deste Reyno del Perú» [1613], en Crónicas peruanas de interés indígena, edición de Francisco Esteve Barba, pp. 279-319. Biblioteca de Autores Españoles, 209. Madrid: Atlas.

Patterson, Thomas C.

1966 «Field Notes: Lurin Valley Project». Manuscrito inédito.

Pollard, A. Mark y Carl Heron

2008 «The Geochemistry of Clays and the Provenance of Ceramics», en Archaeological Chemistry: Second Edition, pp. 98-143. Cambridge: The Royal Society of Chemistry.

PrAdo, Ignacio (ed.)

1974 Crónica moralizada de Antonio de la Calancha. Lima: Universidad Nacional Mayor de San Marcos.

RAMÓN, Gabriel

1999 «Producción alfarera en Santo Domingo de los Olleros (Huarochirí - Lima)». Bulletin de l'Institut Français d'Etudes Andines 28 (2): 215-248.

2011 «The Swallow Potters: Seasonally Migratory Styles in the Andes», en Archaeological Ceramics: A Review of Current Research, Simona Scarcella, ed., pp. 160175. BAR International Series S2193. Oxford: Archaeopress.

Renfrew, Colin

1996 «Prehistory and the Identity of Europe, or don't let's be Beastly to the Hungarians», en Cultural Identity and Archaeology: The Construction of European Communities, Paul Graves-Brown, Siân Jones y Clive Gamble, eds., pp. 125-137. Londres: Routledge.

Rice, Prudence M.

2005 Pottery analysis: a sourcebook. Chicago: University of Chicago Press.

Rostworowski, María

2002 Obras Completas II: Pachacamac. Lima: Instituto de Estudios Peruanos.

SACKETT, James R.

1986 «Isochrestism and Style: A Clarification». Journal of Anthropological Archaeology 5 (3): 266-277.

SAlomon, Frank y Sue Grosboll

2009 «Una visita a los hijos de Chaupi Namca en 1588: desigualdad de género, nombres indígenas y cambios demográficos en el centro de los Andes posincas», en La revisita de Sisicaya, 1588: Huarochirí veinte años antes de Dioses y Hombres, Frank Salomon, Jane Feltham y Sue Grosboll, eds., pp. 17-55. Lima: Pontificia Universidad Católica del Perú. 
SAntillán, Hernando de

1968 «Relación del origen, descendencia, política y gobierno de los incas» [1553], en Crónicas peruanas de interés indígena, edición de Francisco Esteve Barba, pp. 97-149. Biblioteca de Autores Españoles, 209. Madrid: Atlas.

SHENNAN, Stephen

1992 Arqueología cuantitativa. Barcelona: Crítica.

SHIMADA, Izumi

2010 «Un siglo después de Uhle: reflexiones sobre la arqueología de Pachacamac y Perú», en Max Uhle (1856-1944): evaluaciones de sus investigaciones y obras, Peter Kaulicke, Manuela Fisher, Peter Masson y Gregor Wolff, eds., pp. 109-150. Lima: Pontificia Universidad Católica del Perú.

Speakman, Robert J., Michael D. Glascock, Robert H. Tyкot, Christophe Descantes, Jennifer J. Thatcher, Crag E. Skinner y Kyran M. Lienhop

2007 «Selected Applications of Laser Ablation Inductively Coupled Plasma Mass Spectrometry to Archaeological Research», en Archaeological Chemistry, Michael D. Glascock, Robert J. Speakman y Rachel S. Popelka-Filcoff, eds., pp. 275-296. Washngton: American Chemical Society - Oxford University Press.

SpeAKman, Robert J. y Hector NefF

2005 Laser Ablation ICP-MS in Archaeological Research. Albuquerque: University of New Mexico Press.

STARK, Miriam T.

2003 «Current Issues in Ceramic Ethnoarchaeology». Journal of Archaeological Research 11 (3): 193-242.

Strong, William D. y John M. CorbetT

1943 «A Ceramic Sequence at Pachacamac», en Archaeological Studies in Peru, 19411942, William D. Strong, Gordon R. Willey y John M. Corbett, eds., pp. 27-122. Columbia Studies in Archaeology and Ethnology, vol. 1 (3). Nueva York.

Terrádez Gurrea, Manuel

2002 Análisis de componentes principales. Proyecto e-Math, Universitat Oberta de Catalunya. http://www.uoc.edu/in3/emath/docs/Componentes_Principales.pdf, con acceso el 30/9/2013.

TRIGGER, Bruce

1989 A History of Archaeological Thought. Cambridge: Cambridge University Press.

VALlejo, Francisco

2004 «El estilo Ychsma: características generales, secuencia y distribución geográfica». Bulletin de l'Institut Français d'Etudes Andines 33(3): 595-642.

2009 «La problemática de la cerámica Ychsma; El estado de la situación y algunos elementos de discusión». Revista de Antropología 19: 133-168. Santiago de Chile.

VÁzQUEZ DE EsPINOSA, Antonio

1992 Compendio y descripción de las Indias Occidentales [1629], edición de Balbino Velasco Bayón. Madrid: Historia 16. 